\title{
Higher Physical Activity Levels in Children Have Wide Ranging Benefits: Towards Multisectoral Action
}

\author{
Shifalika Goenka ${ }^{1,2 *}$ and RaJi Devarajan ${ }^{1}$ \\ ${ }^{1}$ Centre for Chronic Disease Control, New Delhi, India; ${ }^{2}$ Public Health Foundation of India, Gurugram, Haryana, India. \\ shifalika.goenka@phfi.org
}

I $\mathrm{n}$ this issue of Indian Pediatrics, Mehreen, et al. [1] elicit the validity and reliability of a tool for selfreported physical activity measurement in Indian children and adolescents 10-17 years of age. The correlation coefficients $(95 \% \mathrm{CI})$ for sedentary behaviour and moderate to vigorous physical activity for Modified Physical Activity Questionnaire for Children, [MPAQ(c)], against accelerometer were $0.52(0.36,0.64)$ and $0.41(0.23,0.55)$, respectively, indicating moderate correlation. This is typical of self-reported physical activity tools globally. Self-reported physical activity remains the main stay for population-based studies, due to its low cost and practicality.

The benefits of physical activity are wide-ranging, enormous and lifelong. Also, behaviors etched in childhood set the foundation for adult behaviors. Strong scientific evidence demonstrates that higher amounts of physical activity are associated with increased cardiorespiratory and muscular fitness, improved bone health, cardiometabolic health, academic outcomes, better cognitive development (executive function, attention, memory, crystalized intelligence, processing speed), fewer symptoms of depression, and reduced risk of adiposity in children aged 3 to 17 years [2-5].

The World Health Organisation (WHO) and the Centres for Disease Control and Prevention (CDC) recommend that children and adolescents should indulge in 60 minutes of moderate to vigorous aerobic activity like running or swimming for a minimum of an hour each day. In addition, bone and muscle strengthening activities should be indulged in atleast three days a week. Bone strengthening activities are those which create an impact with gravity signalling the brain towards greater bone strength. Examples are: skipping, jumping, basketball, etc. Muscle strengthening activities include climbing stairs, climbing trees, dancing etc. Every day, several hours of active play is recommended. In addition, it is recommended that children be encouraged towards active transport to school and several hours of play after school hours. Screen time needs to be minimal, if at all, and at the most limited to 2 hours a day [2-5].

Globally, more than $80 \%$ ( $85 \%$ girls and $78 \%$ boys) of school-going adolescents, aged 11-17 years, do not meet the current recommendations of at least one hour of physical activity per day. In India, 73.9\% (71.8\% boys and $76 \%$ girls) of the school going children did not meet the minimum recommended moderate or vigorous physical activity of 60 minutes per day [6]. Inactivity in these school-going adolescents was jeopardizing their present and future health.

"How can we get more children to be more physically active?" is the key public health question. Physical activity should be the most convenient, desirable, safe activity which should be performed during school hours, at home for recreation, and even during daily commute. In the context of the Covid-19 pandemic, open active transport and recreation has taken further precedence. In Europe, huge financial resources have been allocated to further widen the already wide side-walks, and cycling lanes, narrowing motor carriageways to half. Physical activity should be part of an essential foundation of growing up. According to the WHO's Global Action Plan on Physical Activity 2018-2030, we need to create active societies, active environments, active people and active systems [7]. Creating 'active people' requires access to prospects, across myriad settings, to help people of all ages to indulge in regular physical activity as individuals, families and communities. Creating 'active systems' requires implementation of effective synchronized national and subnational action to increase physical activity and reduce sedentary behaviour [7]. Effective governance, leadership, and multisectoral partnerships and advocacy, across all pertinent sectors is the key. Creating 'active environments' requires the establishment and protection of surroundings that promote physical activity in all forms and safeguard the dignity and rights of all people, of all ages, and backgrounds $[7,8]$.

Urban forests/large green lush public parks within 
0.4-0.5 $\mathrm{km}$ radius $(>0.5 \mathrm{Ha})$ of every family, significantly increases physical activity (68-89 minutes or more) [9]. Wide sidewalks as wide as the roads are recommended and so are limiting motor carriageways. Ability to walk safely and pleasurably to school and parks are critical and so are play grounds, and other sporting facilities in schools. Air-pollution should not act as a deterrent to physical activity as the benefits from physical activity outweigh the risks of air pollution [10]. Lush green trees on either side of the road, in schools and in parks not only reduces the ambient temperature, but they also reduce the particulate matter and air-pollution [11]. A 'dashboard' of monitoring and accountability indicators, applicable to the Indian context, pertaining to safety, the environmental factors, accessibility and comfort of physical activity during daily living is required [12].

The Draft National Education Policy (2019) has given an emphasis on incorporating physical education and sports into the curriculum, starting in early school. The National Multi-sectoral Action Plan (NMAP) for prevention and control of common NCDs (2017-2022) in India has placed high importance for promoting physical activity [13]. Khelo India program has been launched by the Ministry of Sports [14]. The 'Fit India' movement was also launched in August, 2019. Dr. Fiona Bull, leading the Division of Physical activity at WHO says "Young people have the right to play and should be provided with the opportunities to realise their right to physical and mental health and well being."

To conclude, 'active children' require active schools, active roads, active policies, active communities, active urban design and active recreation. These will lead to a progressive, equitable, environmentally sensitive and active India.

Funding: SG is partially sponsored by Wellcome Trust Grants (2/8/92/Z/ and 203/24/Z/16/Z) and supported part time by the Centre for Chronic Disease Control. Conflict of interest: None stated.

\section{REFERENCES}

1. Mehreen TS, Ranjani H, Anitha C, Jagannathan N, Pratt M, Mohan V, et al. Reliability and Validity of a Physical Activity Questionnaire for Indian Children and Adolescents. Indian Pediatr. 2020;57:707-11.

2. World Health Organisation. Public consultation on the draft WHO Guidelines on physical activity and sedentary behaviour for children and adolescents, adults and older adults 2020 2020. Available from: https://www.who.int/ news-room/articles-detail/public-consultation-on-thedraft-who-guidelines-on-physical-activity-and-sedentarybehaviour-for-children-and-adolescents-adults-andolder-adults-2020. Accessed on 17 July, 2020.

3. 2018 Physical Activity Guidelines Advisory Committee Scientific Report. Washington, DC: U.S. Department of Health and Human Services, 2018.

4. Australian Institute of Health Welfare. Australia's children 2020. Available from: https://www.aihw.gov.au/ reports/children-youth/australias-children/contents/ health/physical-activity. Accessed on 17 July, 2020.

5. US Department of Health and Human Services. Physical Activity Guidelines for Americans, 2nd Edition. 2019.

6. Guthold R, Stevens GA, Riley LM, Bull FC. Global trends in insufficient physical activity among adolescents: a pooled analysis of 298 population-based surveys with 1.6 million participants. Lancet Child Adolesc Health. 2020;4:23-35.

7. World Health Organisation. Global action plan on physical activity 2018-2030. Geneva, 2018.

8. Heath GW, Parra DC, Sarmiento OL, Andersen LB, Owen $\mathrm{N}$, Goenka S, et al. Evidence-based intervention in physical activity: lessons from around the world. Lancet. 2012;380:272-81.

9. Sallis JF, Cerin E, Conway TL, Adams MA, Frank LD, Pratt $\mathrm{M}$, et al. Physical activity in relation to urban environments in 14 cities worldwide: a cross-sectional study. Lancet. 2016;387:2207-17.

10. Sun S, Cao W, Qiu H, Ran J, Lin H, Shen C, et al. Benefits of physical activity not affected by air pollution: a prospective cohort study. Int J Epidemiol. 2020;49:142-52.

11. Vailshery L, Jaganmohan M, Nagendra H. Effect of street trees on microclimate and air pollution in a tropical city. Urban Forestry and Urban Greening. 2013;12:408-15.

12. Devarajan R, Prabhakaran D, Goenka S. Built environment for physical activity-An urban barometer, surveillance, and monitoring. Obes Rev. 2020;21:e12938.

13. Ministry of Health and Family Welfare GoI. National Multisectoral Action Plan for Prevention and Control of common NCDs (2017-2022) 2017. Available from: https://main.mohfw.gov.in/sites/default/filesNational $\% 20$ Multisectoral\%20Action\%20Plan\%20\%28NMAP\% 29\%20for\%20Prevention\%20and\%20Control\%20of\%20 Common\%20NCDs\%20\%282017-22\%29_1.pdf. Accessed on 17 July, 2020.

14. Ministry of Youth Affairs and Sports GoI. Khelo India 2018. Available from: https://kheloindia.gov.in/about. Accessed on 17 July, 2020. 